\title{
Two New Approximations for Variable-Order Fractional Derivatives
}

\author{
Ruilian Du and Zongqi Liang \\ School of Sciences, Jimei University, Xiamen, Fujian 361021, China \\ Correspondence should be addressed to Zongqi Liang; zqliang@jmu.edu.cn
}

Received 15 May 2017; Accepted 2 July 2017; Published 31 July 2017

Academic Editor: Jorge E. Macías-Díaz

Copyright (C) 2017 Ruilian Du and Zongqi Liang. This is an open access article distributed under the Creative Commons Attribution License, which permits unrestricted use, distribution, and reproduction in any medium, provided the original work is properly cited.

We introduced a parameter $\sigma(t)$ which was related to $\alpha(t)$; then two numerical schemes for variable-order Caputo fractional derivatives were derived; the second-order numerical approximation to variable-order fractional derivatives $\alpha(t) \in(0,1)$ and $3-\alpha(t)$-order approximation for $\alpha(t) \in(1,2)$ are established. For the given parameter $\sigma(t)$, the error estimations of formulas were proven, which were higher than some recently derived schemes. Finally, some numerical examples with exact solutions were studied to demonstrate the theoretical analysis and verify the efficiency of the proposed methods.

\section{Introduction}

Fractional differential equations include constant-order and variable-order equations; a great quantity of natural phenomena can be modeled by variable-order fractional differential equations; the study of such problems has attracted much attention. In recent years, profound background of physical applications for the variable-order fractional calculus has been already established; the definition of variable-order operator has been investigated in [1-4]. Numerous problems in mathematical physics and engineering have been modeled by variable-order fractional differential equations, such as successful applications in mechanics [5], in the simulation of linear and nonlinear viscoelasticity oscillators [6], and in other cases where the order of the derivative varies with time [7]. In addition, a physical experimental investigation of variable-order operators has been considered in [8]. Variable-order fractional derivatives can be used to model anomalous diffusion, as they can describe the time dependent diffusion process more specifically than fractional derivatives of constant order, just as shown in [9]. The difference schemes of fractional derivatives with constant and variable order are investigated in [10].

Due to the existence of variable fractional derivative, it is usually difficult to obtain the analytical solution of such equations; therefore, it is particularly significant to give numerical solutions to these problems. Cao and Qiu [11] derived a high order numerical method for variable-order fractional ordinary differential equation by establishing a second-order numerical approximation to variable-order Riemann-Liouville fractional derivative. Fu et al. [12] adopted the method of approximate particular solutions for both constant-order and variable-order time fractional diffusion models. Several finite difference methods for variable-order fractional partial diffusion equations were proposed in [13$18]$.

As what we can see above, there are many different definitions of the variable-order fractional derivatives. However, different from other definitions in mathematics, only initial condition is needed for the variable-order Caputo definition which can be easily used in physical field; this definition means that the memory rate of system is determined by the current time instant and changes with time. For simplicity, only variable-order Caputo definition was discussed in the whole paper.

A good approximation of the variable-order Caputo derivatives was observed in [19], it was defined at the points $t_{n+1 / 2}$ and $t_{n}$; then what will happen if it is redefined in a neighborhood at the point $t_{n}$ ? Approximation formula for the $\alpha$-order $(0 \leq \alpha \leq 1)$ Caputo derivatives at the point $t=t_{n-1+\sigma}$ was investigated in [20], where $\sigma=1-\alpha / 2$ is a superconvergence point which ensures that the convergence order improves from the local second order to the overall $3-\alpha$ order. In the current work, we applied the ideas in [20]; a 
parameter $\sigma(t)$ was given, which was changed with $\alpha(t)$, and the selection of $\sigma(t)$ played a vital role in the numerical results in the paper. We proposed two new approximation formulas of second-order and $3-\alpha(t)$ accuracy for variable-order time fractional operator with orders $0<\alpha(t)<1$ and $1<\alpha(t)<2$, respectively. Specifically, we adopted the following definition of variable-order Caputo fractional derivatives:

$$
\begin{array}{r}
{ }_{0}^{C} D_{t}^{\alpha(t)} f(t)=\frac{1}{\Gamma(n-\alpha(t))} \int_{0}^{t} \frac{f^{(n)}(s)}{(t-s)^{\alpha(t)-n+1}} d s, \\
n-1<\alpha(t)<n .
\end{array}
$$

The paper is organized as follows. In the next section, we present two new formulas, select $\sigma(t)=1-\alpha(t) / 2$ for $0<\alpha(t)<1$ and $\sigma(t)=3 / 2-\alpha(t) / 2$ for $1<\alpha(t)<2$, respectively, and provide the corresponding error analysis. In Section 3, the numerical verifications are presented; as can be seen from the numerical results, minor changes to the selected parameters $\sigma(t)$ will have a significant impact on the error estimates. Finally, in Section 4, the conclusion is drawn.

\section{Approximation Formulas}

In this section, we present the following lemma, which plays a vital role in the later analysis.

Lemma 1 (see [19]). Assuming that the derivatives of the function $f(x)$ exist to the order of $n$ on $[a, b]$ and to the order of $n+1$ on $(a, b), a \leq x_{0}<x_{1}<\cdots<x_{n} \leq b, p_{n}(x)$ is the $n$th degree interpolation polynomial of $f(x)$ based on the points $x_{0}, x_{1}, \ldots, x_{n}$, then, for $x \in[a, b]$, the following holds:

$$
\begin{array}{r}
f^{(k)}(x)-p_{n}^{(k)}(x) \\
=\frac{f^{(n+1)}(\xi)}{(n-k+1) !}\left(x-x_{0}^{(k)}\right)\left(x-x_{1}^{(k)}\right) \cdots\left(x-x_{n-k}^{(k)}\right), \\
\quad(k=0,1,2, \ldots),
\end{array}
$$

where $\xi \in(a, b)$ depends on $k$ and $x$, and $x_{i}<x_{i}^{(k)}<x_{i+k}(i=$ $0,1, \ldots, n-k)$.

For a given function $f(x)$ and an integer $N>0$, denote $t_{n}=n \tau, f^{n}=f\left(t_{n}\right), n=0,1, \ldots, N$, where $\tau=T / N$ is the step. Introduce the following notation:

$$
\begin{aligned}
t_{n+\sigma} & =(n+\sigma) \tau, \\
\delta_{t} f^{n+1 / 2} & =\frac{1}{\tau}\left(f^{n+1}-f^{n}\right), \\
\delta_{t}^{2} f^{n} & =\frac{\delta_{t} f^{n+1 / 2}-\delta_{t} f^{n-1 / 2}}{\tau},
\end{aligned}
$$

and, for convenience, denote $\sigma=\sigma\left(t_{n}\right)$.

2.1. Second-Order Formula for $\alpha(t) \in(0,1)$. Denote $\alpha_{n-1+\sigma}=$ $\alpha\left(t_{n-1+\sigma}\right), \sigma=1-\alpha\left(t_{n-1+\sigma}\right) / 2, n=0,1, \ldots, N-1$.
Estimating the derivative ${ }_{0}^{C} D_{t}^{\alpha(t)} f(t)$ with order $\alpha(t) \epsilon$ $(0,1)$ at the grid point $t_{n-1+\sigma}, n=0,1, \ldots, N-1$, from (1), we directly obtain

$$
\begin{aligned}
& { }_{0}^{C} D_{t}^{\alpha_{n-1+\sigma}} f\left(t_{n-1+\sigma}\right) \\
& =\frac{1}{\Gamma\left(1-\alpha_{n-1+\sigma}\right)} \int_{0}^{t_{n-1+\sigma}} \frac{f^{\prime}(s)}{\left(t_{n-1+\sigma}-s\right)^{\alpha_{n-1+\sigma}}} d s .
\end{aligned}
$$

Evaluate the integration on each subinterval, leading to

$$
\begin{aligned}
& { }_{0}^{C} D_{t}^{\alpha_{n-1+\sigma}} f\left(t_{n-1+\sigma}\right) \\
& =\frac{1}{\Gamma\left(1-\alpha_{n-1+\sigma}\right)}\left[\sum_{k=1}^{n-1} \int_{t_{k-1}}^{t_{k}} \frac{f^{\prime}(s)}{\left(t_{n-1+\sigma}-s\right)^{\alpha_{n-1+\sigma}}} d s\right. \\
& \left.\quad+\int_{t_{n-1}}^{t_{n-1+\sigma}} \frac{f^{\prime}(s)}{\left(t_{n-1+\sigma}-s\right)^{\alpha_{n-1+\sigma}}} d s\right] .
\end{aligned}
$$

For each interval $\left[t_{k-1}, t_{k}\right], k=1, \ldots, n-1$, denote the second-degree interpolation polynomial in the Lagrange form as follows:

$$
\begin{aligned}
L_{k}^{2} f(s)= & \frac{f^{k-1}\left(t-t_{k}\right)\left(t-t_{k+1}\right)}{2 \tau^{2}} \\
& -\frac{f^{k}\left(t-t_{k-1}\right)\left(t-t_{k+1}\right)}{\tau^{2}} \\
& +\frac{f^{k+1}\left(t-t_{k-1}\right)\left(t-t_{k}\right)}{2 \tau^{2}},
\end{aligned}
$$

and for the last interval $\left[t_{n-1}, t_{n-1+\sigma}\right]$, denote the first-degree interpolation polynomial in the Lagrange form as

$$
L_{n}^{1} f(s)=-\frac{f^{n-1}\left(t-t_{n}\right)}{\tau}+\frac{f^{n}\left(t-t_{n-1}\right)}{\tau} .
$$

As an approximation formula, the following result is obtained:

$$
\begin{aligned}
f(s)=L_{k}^{2} f(s)+r_{k}(s), & \\
& s \in\left[t_{k-1}, t_{k}\right], k=1, \ldots, n-1,
\end{aligned}
$$

where

$$
\begin{aligned}
& r_{k}(s)=\frac{f^{\prime \prime \prime}\left(\xi_{k}\right)}{6}\left(s-t_{k-1}\right)\left(s-t_{k}\right)\left(s-t_{k+1}\right), \\
& \xi_{k} \in\left(t_{k-1}, t_{k+1}\right), \\
& f(s)=L_{n}^{1} f(s)+r_{n}(s), \quad s \in\left[t_{n-1}, t_{n-1+\sigma}\right]
\end{aligned}
$$

where

$$
r_{n}(s)=\frac{f^{\prime \prime}\left(\xi_{n}\right)}{2}\left(s-t_{n-1}\right)\left(s-t_{n}\right), \quad \xi_{n} \in\left(t_{n-1}, t_{n}\right) .
$$


Substituting (8) and (10) into (5) yields

$$
\begin{aligned}
& { }_{0}^{C} D_{t}^{\alpha_{n-1+\sigma}} f\left(t_{n-1+\sigma}\right) \\
& =\frac{1}{\Gamma\left(1-\alpha_{n-1+\sigma}\right)}\left[\sum_{k=1}^{n-1} \int_{t_{k-1}}^{t_{k}} \frac{\left(L_{k}^{2} f(s)\right)^{\prime}}{\left(t_{n-1+\sigma}-s\right)^{\alpha_{n-1+\sigma}}} d s\right. \\
& \left.\quad+\int_{t_{n-1}}^{t_{n-1+\sigma}} \frac{\left(L_{n}^{1} f(s)\right)^{\prime}}{\left(t_{n-1+\sigma}-s\right)^{\alpha_{n-1+\sigma}}} d s\right]+R^{n-1+\sigma},
\end{aligned}
$$

and the truncation error is

$$
\begin{aligned}
& R^{n-1+\sigma}=\frac{1}{\Gamma\left(1-\alpha_{n-1+\sigma}\right)}\left[\sum_{k=1}^{n-1} \int_{t_{k-1}}^{t_{k}} \frac{r_{k}^{\prime}(s)}{\left(t_{n-1+\sigma}-s\right)^{\alpha_{n-1+\sigma}}} d s\right. \\
& \left.\quad+\int_{t_{n-1}}^{t_{n-1+\sigma}} \frac{r_{n}^{\prime}(s)}{\left(t_{n-1+\sigma}-s\right)^{\alpha_{n-1+\sigma}}} d s\right] .
\end{aligned}
$$

Here we denote the discrete approximation formula for the variable-order derivative with order $\alpha_{n-1+\sigma}$ as ${ }_{0} \Delta_{t}^{\alpha_{n-1+\sigma}} f^{n-1+\sigma}$; thus from (12), it is easy to get the following result:

$$
\begin{aligned}
& { }_{0} \Delta_{t}^{\alpha_{n-1+\sigma}} f^{n-1+\sigma}=\frac{1}{\Gamma\left(1-\alpha_{n-1+\sigma}\right)}\left[\sum_{k=1}^{n-1} \int_{t_{k-1}}^{t_{k}} \frac{\left(L_{k}^{2} f(s)\right)^{\prime}}{\left(t_{n-1+\sigma}-s\right)^{\alpha_{n-1+\sigma}}} d s+\int_{t_{n-1}}^{t_{n-1+\sigma}} \frac{\left(L_{n}^{1} f(s)\right)^{\prime}}{\left(t_{n-1+\sigma}-s\right)^{\alpha_{n-1+\sigma}}} d s\right] \\
& =\frac{1}{\Gamma\left(1-\alpha_{n-1+\sigma}\right)}\left[\sum_{k=1}^{n-1} \int_{t_{k-1}}^{t_{k}} \frac{(1 / 2)\left(\delta_{t} f^{k+1 / 2}+\delta_{t} f^{k-1 / 2}\right)+\delta_{t}^{2} f^{k}\left(s-t_{k}\right)}{\left(t_{n-1+\sigma}-s\right)^{\alpha_{n-1+\sigma}}} d s+\int_{t_{n-1}}^{t_{n-1+\sigma}} \frac{\delta_{t} f^{n-1 / 2}}{\left(t_{n-1+\sigma}-s\right)^{\alpha_{n-1+\sigma}}} d s\right] \\
& =\frac{1}{\Gamma\left(1-\alpha_{n-1+\sigma}\right)}\left\{\sum_{k=1}^{n-1} \frac{\left(\delta_{t} f^{k+1 / 2}+\delta_{t} f^{k-1 / 2}\right) \tau^{1-\alpha_{n-1+\sigma}}}{2\left(1-\alpha_{n-1+\sigma}\right)}\left[(n+\sigma-k)^{1-\alpha_{n-1+\sigma}}-(n+\sigma-k-1)^{1-\alpha_{n-1+\sigma}}\right]\right. \\
& \left.+\frac{\delta_{t} f^{n-1 / 2} \tau^{1-\alpha_{n-1+\sigma}}}{1-\alpha_{n-1+\sigma}} \sigma^{1-\alpha_{n-1+\sigma}}+\sum_{k=1}^{n-1} \int_{t_{k-1}}^{t_{k}} \frac{\delta_{t}^{2} f^{k}\left(s-t_{k}\right)}{\left(t_{n-1+\sigma}-s\right)^{\alpha_{n-1+\sigma}}} d s\right\} \\
& =\frac{\tau^{1-\alpha_{n-1+\sigma}}}{\Gamma\left(2-\alpha_{n-1+\sigma}\right)}\left\{\sum_{k=1}^{n-1} \frac{\left(\delta_{t} f^{k+1 / 2}+\delta_{t} f^{k-1 / 2}\right)}{2}\left[(n+\sigma-k)^{1-\alpha_{n-1+\sigma}}-(n+\sigma-k-1)^{1-\alpha_{n-1+\sigma}}\right]\right. \\
& \left.+\sum_{k=1}^{n-1} \delta_{t}^{2} f^{k} \tau\left[\frac{1}{2-\alpha_{n-1+\sigma}}\left((n+\sigma-k)^{2-\alpha_{n-1+\sigma}}-(n+\sigma-k-1)^{2-\alpha_{n-1+\sigma}}\right)-(n+\sigma-k)^{1-\alpha_{n-1+\sigma}}\right]+\delta_{t} f^{n-1 / 2} \sigma^{1-\alpha_{n-1+\sigma}}\right\} \\
& =\frac{\tau^{1-\alpha_{n-1+\sigma}}}{\Gamma\left(2-\alpha_{n-1+\sigma}\right)}\left[\sum_{k=1}^{n-1} a_{n-k}^{\left(\alpha_{n-1+\sigma}\right)}\left(\delta_{t} f^{k+1 / 2}+\delta_{t} f^{k-1 / 2}\right)+\sum_{k=1}^{n-1} b_{n-k}^{\left(\alpha_{n-1+\sigma}\right)} \tau \delta_{t}^{2} f^{k}+\delta_{t} f^{n-1 / 2} \sigma^{1-\alpha_{n-1+\sigma}}\right] \\
& =\frac{\tau^{1-\alpha_{n-1+\sigma}}}{\Gamma\left(2-\alpha_{n-1+\sigma}\right)}\left[\sum_{k=1}^{n-1} a_{n-k}^{\left(\alpha_{n-1+\sigma}\right)}\left(\delta_{t} f^{k+1 / 2}+\delta_{t} f^{k-1 / 2}\right)+\sum_{k=1}^{n-1} b_{n-k}^{\left(\alpha_{n-1+\sigma}\right)}\left(\delta_{t} f^{k+1 / 2}-\delta_{t} f^{k-1 / 2}\right)+\delta_{t} f^{n-1 / 2} \sigma^{1-\alpha_{n-1+\sigma}}\right] \\
& =\frac{\tau^{1-\alpha_{n-1+\sigma}}}{\Gamma\left(2-\alpha_{n-1+\sigma}\right)}\left[\sum_{k=2}^{n-1}\left(a_{n-k+1}^{\left(\alpha_{n-1+\sigma}\right)}+a_{n-k}^{\left(\alpha_{n-1+\sigma}\right)}+b_{n-k+1}^{\left(\alpha_{n-1+\sigma}\right)}-b_{n-k}^{\left(\alpha_{n-1+\sigma}\right)}\right) \delta_{t} f^{k-1 / 2}+\left(a_{1}^{\left(\alpha_{n-1+\sigma}\right)}+a_{0}^{\left(\alpha_{n-1+\sigma}\right)}+b_{1}^{\left(\alpha_{n-1+\sigma}\right)}\right) \delta_{t} f^{n-1 / 2}\right. \\
& \left.+\left(a_{n-1}^{\left(\alpha_{n-1+\sigma}\right)}-b_{n-1}^{\left(\alpha_{n-1+\sigma}\right)}\right) \delta_{t} f^{1 / 2}\right]=\frac{\tau^{1-\alpha_{n-1+\sigma}}}{\Gamma\left(2-\alpha_{n-1+\sigma}\right)} \sum_{k=1}^{n} d_{n-k}^{\left(\alpha_{n-1+\sigma}\right)} \delta_{t} f^{k-1 / 2}=\frac{\tau^{-\alpha_{n-1+\sigma}}}{\Gamma\left(2-\alpha_{n-1+\sigma}\right)} \sum_{k=1}^{n} d_{n-k}^{\left(\alpha_{n-1+\sigma}\right)}\left(f^{k}-f^{k-1}\right),
\end{aligned}
$$

where

$$
\begin{aligned}
& a_{0}^{\left(\alpha_{n-1+\sigma}\right)}=\sigma^{1-\alpha_{n-1+\sigma},} \\
& a_{k}^{\left(\alpha_{n-1+\sigma}\right)}=\frac{1}{2}\left[(k+\sigma)^{1-\alpha_{n-1+\sigma}}-(k+\sigma-1)^{1-\alpha_{n-1+\sigma}}\right], \\
& b_{k}^{\left(\alpha_{n-1+\sigma}\right)} \quad 1 \leq k \leq n-1, \\
& =\frac{1}{2-\alpha_{n-1+\sigma}}\left[(k+\sigma)^{2-\alpha_{n-1+\sigma}}-(k+\sigma-1)^{2-\alpha_{n-1+\sigma}}\right]
\end{aligned}
$$$$
-(k+\sigma)^{1-\alpha_{n-1+\sigma}}, \quad 1 \leq k \leq n-1 .
$$$$
d_{k}^{\left(\alpha_{n-1+\sigma}\right)}
$$$$
= \begin{cases}a_{1}^{\left(\alpha_{n-1+\sigma}\right)}+a_{0}^{\left(\alpha_{n-1+\sigma}\right)}+b_{1}^{\left(\alpha_{n-1+\sigma}\right)}, & k=0, \\ a_{k+1}^{\left(\alpha_{n-1+\sigma}\right)}+a_{k}^{\left(\alpha_{n-1+\sigma}\right)}+b_{k+1}^{\left(\alpha_{n-1+\sigma}\right)}-b_{k}^{\left(\alpha_{n-1+\sigma}\right)}, & 1 \leq k \leq n-2, \\ a_{k}^{\left(\alpha_{n-1+\sigma}\right)}-b_{k}^{\left(\alpha_{n-1+\sigma}\right)}, & k=n-1 .\end{cases}
$$ 
Next, the analysis for the approximation error of formula (12) was given in detail.

Theorem 2. Let $\alpha_{n-1+\sigma} \in(0,1), f \in C^{3}\left(\left[0, t_{n+1}\right]\right)$; the following holds:

$$
\begin{gathered}
\left|{ }_{0}^{C} D_{t}^{\alpha_{n-1+\sigma}} f\left(t_{n-1+\sigma}\right)-{ }_{0} \Delta_{t}^{\alpha_{n-1+\sigma}} f^{n-1+\sigma}\right| \\
\leq \frac{1}{\Gamma\left(1-\alpha_{n-1+\sigma}\right)}\left[\Theta_{n_{t_{0} \leq t \leq t_{n-1}}} \max \left|f^{\prime \prime}(t)\right|\right. \\
\left.\quad+\frac{\sigma^{1-\alpha_{n-1+\sigma}} \tau^{-\alpha_{n-1+\sigma}}}{1-\alpha_{n-1+\sigma}} \max _{t_{n-1} \leq t \leq t_{n}}\left|f^{\prime \prime}(t)\right|\right] \tau^{2},
\end{gathered}
$$

where $\Theta_{1}=\tau^{-\alpha_{\sigma}} \sigma^{1-\alpha_{\sigma}} /\left(1-\alpha_{\sigma}\right), \Theta_{n}=c_{1} t_{n-1+\sigma}^{1-\alpha_{n-1+\sigma}} /(1-$ $\left.\alpha_{n-1+\sigma}\right), n=2, \ldots, N-1, c_{1}$ is a positive constant.

Proof. From (12), obviously

$$
\begin{aligned}
& R^{n-1+\sigma}=\frac{1}{\Gamma\left(1-\alpha_{n-1+\sigma}\right)}\left[\sum_{k=1}^{n-1} \int_{t_{k-1}}^{t_{k}} \frac{r_{k}^{\prime}(s)}{\left(t_{n-1+\sigma}-s\right)^{\alpha_{n-1+\sigma}}} d s\right. \\
& \left.\quad+\int_{t_{n-1}}^{t_{n-1+\sigma}} \frac{r_{n}^{\prime}(s)}{\left(t_{n-1+\sigma}-s\right)^{\alpha_{n-1+\sigma}}} d s\right] \\
& \quad=\frac{1}{\Gamma\left(1-\alpha_{n-1+\sigma}\right)}\left(R_{1}+R_{2}\right) .
\end{aligned}
$$

Using Lemma 1, it produces

$$
\begin{aligned}
& r_{k}^{\prime}(s)=\frac{1}{2} f^{\prime \prime \prime}\left(\eta_{k}\right)\left(s-t_{0}^{(k)}\right)\left(s-t_{1}^{(k)}\right), \\
& \eta_{k} \in\left(t_{k-1}, t_{k+1}\right), t_{0}^{(k)} \in\left(t_{k-1}, t_{k}\right), t_{1}^{(k)} \in\left(t_{k}, t_{k+1}\right), \quad 1 \leq k \leq n-1, \\
& r_{n}^{\prime}(s)=f^{\prime \prime}\left(\eta_{n}\right)\left(s-t_{0}^{(n)}\right), \quad \eta_{n} \in\left(t_{n-1}, t_{n}\right), t_{0}^{(n)} \in\left(t_{n-1}, t_{n}\right) .
\end{aligned}
$$

Next, the error on each interval is analyzed.

When $n=1$, from (19), it follows that

$$
\begin{aligned}
\left|\int_{0}^{t_{\sigma}} \frac{r_{1}^{\prime}(s)}{\left(t_{\sigma}-s\right)^{\alpha_{\sigma}}} d s\right| & \leq \tau \max _{t_{0} \leq t \leq t_{1}}\left|f^{\prime \prime}(t)\right| \int_{0}^{t_{\sigma}} \frac{1}{\left(t_{\sigma}-s\right)^{\alpha_{\sigma}}} d s \\
& =\frac{\tau^{-\alpha_{\sigma}} \sigma^{1-\alpha_{\sigma}}}{1-\alpha_{\sigma}} \max _{t_{0} \leq t \leq t_{1}}\left|f^{\prime \prime}(t)\right| \tau^{2} .
\end{aligned}
$$

When $n=2, \ldots, N-1$, from (18), this leads to the following estimation:

$$
\begin{aligned}
\left|R_{1}\right| & =\left|\sum_{k=1}^{n-1} \int_{t_{k-1}}^{t_{k}} \frac{r_{k}^{\prime}(s)}{\left(t_{n-1+\sigma}-s\right)^{\alpha_{n-1+\sigma}}} d s\right| \\
& =\left|\sum_{k=1}^{n-1} \frac{1}{2} \int_{t_{k-1}}^{t_{k}} \frac{f^{\prime \prime \prime}\left(\eta_{k}\right)\left(s-t_{0}^{(k)}\right)\left(s-t_{1}^{(k)}\right)}{\left(t_{n-1+\sigma}-s\right)^{\alpha_{n-1+\sigma}}} d s\right| \\
& \leq c_{1} \max _{t_{0} \leq t \leq t_{n-1}}\left|f^{\prime \prime \prime}(t)\right|\left|\int_{t_{0}}^{t_{n-1}} \frac{1}{\left(t_{n-1+\sigma}-s\right)^{\alpha_{n-1+\sigma}}} d s\right| \tau^{2} \\
& =\frac{c_{1} t_{n-1+\sigma}^{1-\alpha_{n-1+\sigma}}}{1-\alpha_{n-1+\sigma}} \max _{0} \leq t \leq t_{n-1}
\end{aligned}
$$

where $c_{1}$ is a positive constant.
From (19), it can be obtained in the same method.

$$
\begin{aligned}
\left|R_{2}\right| & =\left|\int_{t_{n-1}}^{t_{n-1+\sigma}} \frac{r_{n}^{\prime}(s)}{\left(t_{n-1+\sigma}-s\right)^{\alpha_{n-1+\sigma}}} d s\right| \\
& \leq \tau \max _{t_{n-1} \leq t \leq t_{n}}\left|f^{\prime \prime}(t)\right|\left|\int_{t_{n-1}}^{t_{n-1+\sigma}} \frac{1}{\left(t_{n-1+\sigma}-s\right)^{\alpha_{n-1+\sigma}}} d s\right| \\
& =\frac{\sigma^{1-\alpha_{n-1+\sigma}} \tau^{-\alpha_{n-1+\sigma}}}{1-\alpha_{n-1+\sigma}} \max _{t_{n-1} \leq t \leq t_{n}}\left|f^{\prime \prime}(t)\right| \tau^{2} .
\end{aligned}
$$

Substituting (20)-(22) into (17) leads to the theorem.

2.2. Second-Order Formula for $\alpha(t) \in(1,2)$. Denote $f_{0}^{\prime}=$ $f^{\prime}\left(t_{0}\right), \sigma=3 / 2-\alpha_{n-1+\sigma} / 2$. Computing the variable-order derivative with order $\alpha(t)(1<\alpha(t)<2)$ at $t_{n-1+\sigma}$,

$$
\begin{aligned}
& { }_{0}^{C} D_{t}^{\alpha_{n-1+\sigma}} f\left(t_{n-1+\sigma}\right)=\frac{1}{\Gamma\left(2-\alpha_{n-1+\sigma}\right)} \\
& \cdot \int_{t_{0}}^{t_{n-1+\sigma}} \frac{f^{\prime \prime}(s)}{\left(t_{n-1+\sigma}-s\right)^{\alpha_{n-1+\sigma}-1}} d s \\
& =\frac{1}{\Gamma\left(2-\alpha_{n-1+\sigma}\right)}\left[\int_{t_{0}}^{t_{1}} \frac{f^{\prime \prime}(s)}{\left(t_{n-1+\sigma}-s\right)^{\alpha_{n-1+\sigma}-1}} d s\right. \\
& +\sum_{j=1}^{n-2} \int_{t_{j}}^{t_{j+1}} \frac{f^{\prime \prime}(s)}{\left(t_{n-1+\sigma}-s\right)^{\alpha_{n-1+\sigma}-1}} d s \\
& \left.+\int_{t_{n-1}}^{t_{n-1+\sigma}} \frac{f^{\prime \prime}(s)}{\left(t_{n-1+\sigma}-s\right)^{\alpha_{n-1+\sigma}-1}} d s\right] .
\end{aligned}
$$

For the interval $\left[t_{0}, t_{1}\right]$, the cubic interpolation polynomial in the Hermite form is as

$$
\begin{aligned}
& L_{0}^{3} f(s)=f^{0}+f_{0}^{\prime}\left(s-t_{0}\right)+\frac{(1 / \tau)\left(f^{1}-f^{0}\right)-f_{0}^{\prime}}{\tau}(s \\
& \left.-t_{0}\right)^{2}+\frac{1}{2 \tau}\left[\frac{(1 / \tau)\left(f^{1}-f^{0}\right)-f_{0}^{\prime}}{\tau}\right. \\
& \left.-\frac{(1 / \tau)\left(f^{2}-f^{1}\right)-(1 / \tau)\left(f^{1}-f^{0}\right)}{2 \tau}\right]\left(s-t_{0}\right)^{2}(s \\
& \left.-t_{1}\right),
\end{aligned}
$$

and it follows that

$$
f(s)=L_{0}^{3} f(s)+r_{0}(s)
$$

the truncation error is

$$
r_{0}(s)=\frac{f^{(4)}\left(\xi_{0}\right)}{24}\left(s-t_{0}\right)^{2}\left(s-t_{1}\right)\left(s-t_{2}\right),
$$


For the interval $\left[t_{j}, t_{j+1}\right], j=1, \ldots, n-2$, the cubic interpolation polynomial at points $t_{j-1}, t_{j}, t_{j+1}$, and $t_{j+2}$ in the Lagrange form is as

$$
L_{j}^{3} f(s)=\sum_{k=j-1}^{j+2} f^{k} \prod_{m=j-1, m \neq k}^{j+2} \frac{s-t_{m}}{t_{k}-t_{m}},
$$

leading to

$$
f(s)=L_{j}^{3} f(s)+r_{j}(s)
$$

the truncation error is

$$
r_{j}(s)=\frac{f^{(4)}\left(\xi_{j}\right)}{24} \prod_{k=-1}^{2}\left(s-t_{k+j}\right), \quad \xi_{j} \in\left(t_{j-1}, t_{j+2}\right) ;
$$

using the cubic interpolation polynomial at points $t_{n-2}, t_{n-1}$, and $t_{n}$ in the Lagrange form to approximate $f(s)$ on the last interval $\left[t_{n-1}, t_{n-1+\sigma}\right]$, we get

$$
\begin{aligned}
L_{n}^{2} f(s)= & \frac{f^{n-2}\left(t-t_{n-1}\right)\left(t-t_{n}\right)}{2 \tau^{2}} \\
& -\frac{f^{n-1}\left(t-t_{n-2}\right)\left(t-t_{n}\right)}{\tau^{2}} \\
& +\frac{f^{n}\left(t-t_{n-2}\right)\left(t-t_{n-1}\right)}{2 \tau^{2}},
\end{aligned}
$$

the following holds:

$$
f(s)=L_{n}^{2} f(s)+r_{n}(s) ;
$$

the truncation error is

$$
\begin{array}{r}
r_{n}(s)=\frac{f^{\prime \prime \prime}\left(\xi_{n}\right)}{6}\left(s-t_{n-2}\right)\left(s-t_{n-1}\right)\left(s-t_{n}\right), \\
\xi_{n} \in\left(t_{n-2}, t_{n}\right) .
\end{array}
$$

Substituting (25), (28), and (31) into (23), there is

$$
\begin{aligned}
& { }_{0}^{C} D_{t}^{\alpha_{n-1+\sigma}} f\left(t_{n-1+\sigma}\right) \\
& =\frac{1}{\Gamma\left(2-\alpha_{n-1+\sigma}\right)}\left[\int_{t_{0}}^{t_{1}} \frac{\left(L_{0}^{3} f(s)+r_{0}(s)\right)^{\prime \prime}}{\left(t_{n-1+\sigma}-s\right)^{\alpha_{n-1+\sigma}-1}} d s\right.
\end{aligned}
$$

$$
\begin{aligned}
& +\sum_{j=1}^{n-2} \int_{t_{j}}^{t_{j+1}} \frac{\left(L_{j}^{3} f(s)+r_{j}(s)\right)^{\prime \prime}}{\left(t_{n-1+\sigma}-s\right)^{\alpha_{n-1+\sigma}-1}} d s \\
& \left.+\int_{t_{n-1}}^{t_{n-1+\sigma}} \frac{\left(L_{n}^{2} f(s)+r_{n}(s)\right)^{\prime \prime}}{\left(t_{n-1+\sigma}-s\right)^{\alpha_{n-1+\sigma}-1}} d s\right] .
\end{aligned}
$$

Denote the approximation formula of the variable-order derivative with order $\alpha_{n-1+\sigma}$ as ${ }_{0} \Delta_{t}^{\alpha_{n-1+\sigma}} f^{n-1+\sigma}$ and obtain

$$
\begin{aligned}
& { }_{0} \Delta_{t}^{\alpha_{n-1+\sigma}} f^{n-1+\sigma} \\
& =\frac{1}{\Gamma\left(2-\alpha_{n-1+\sigma}\right)}\left[\sum_{j=0}^{n-2} \int_{t_{j}}^{t_{j+1}} \frac{\left(L_{j}^{3} f(s)\right)^{\prime \prime}}{\left(t_{n-1+\sigma}-s\right)^{\alpha_{n-1+\sigma}-1}} d s\right. \\
& \left.\quad+\int_{t_{n-1}}^{t_{n-1+\sigma}} \frac{\left(L_{n}^{2} f(s)\right)^{\prime \prime}}{\left(t_{n-1+\sigma}-s\right)^{\alpha_{n-1+\sigma}-1}} d s\right] \\
& =\frac{1}{\Gamma\left(2-\alpha_{n-1+\sigma}\right)}\left[\int_{t_{0}}^{t_{1}} \frac{\left(L_{0}^{3} f(s)\right)^{\prime \prime}}{\left(t_{n-1+\sigma}-s\right)^{\alpha_{n-1+\sigma}-1}} d s\right. \\
& \quad+\sum_{j=1}^{n-2} \int_{t_{j}}^{t_{j+1}} \frac{\left(L_{j}^{3} f(s)\right)^{\prime \prime}}{\left(t_{n-1+\sigma}-s\right)^{\alpha_{n-1+\sigma}-1}} d s \\
& \left.\quad+\int_{t_{n-1}}^{t_{n-1+\sigma}} \frac{\left(L_{n}^{2} f(s)\right)^{\prime \prime}}{\left(t_{n-1+\sigma}-s\right)^{\alpha_{n-1+\sigma}-1}} d s\right],
\end{aligned}
$$

where

$$
\begin{aligned}
&\left(L_{0}^{3} f(s)\right)^{\prime \prime}= \frac{s-t_{0}}{\tau} \frac{f^{2}-2 f^{1}+f^{0}}{\tau^{2}} \\
&+\frac{t_{1}-s}{\tau} \frac{8 f^{1}-f^{2}-7 f^{0}-6 \tau f_{0}^{\prime}}{2 \tau^{2}}, \\
&\left(L_{j}^{3} f(s)\right)^{\prime \prime}= \frac{t_{j+1}-s}{\tau} \frac{f^{j-1}-2 f^{j}+f^{j+1}}{\tau^{2}} \\
&+\frac{s-t_{j}}{\tau} \frac{f^{j}-2 f^{j+1}+f^{j+2}}{\tau^{2}}, \\
&\left(L_{n}^{2} f(s)\right)^{\prime \prime}= \delta_{t}^{2} f^{n-1} . \\
& \text { Substituting the above formula into (34), thus }
\end{aligned}
$$

$$
\begin{aligned}
& { }_{0} \Delta_{t}^{\alpha_{n-1+\sigma}} f^{n-1+\sigma} \\
& \quad=\frac{1}{\Gamma\left(2-\alpha_{n-1+\sigma}\right)}\left[\int_{t_{0}}^{t_{1}} \frac{\left(\left(s-t_{0}\right) / \tau\right)\left(\left(f^{2}-2 f^{1}+f^{0}\right) / \tau^{2}\right)+\left(\left(t_{1}-s\right) / \tau\right)\left(\left(8 f^{1}-f^{2}-7 f^{0}-6 \tau f_{0}^{\prime}\right) / 2 \tau^{2}\right)}{\left(t_{n-1+\sigma}-s\right)^{\alpha_{n-1+\sigma}-1}} d s\right.
\end{aligned}
$$




$$
\begin{aligned}
& +\sum_{j=1}^{n-2} \int_{t_{j}}^{t_{j+1}} \frac{\left(\left(t_{j+1}-s\right) / \tau\right)\left(\left(f^{j-1}-2 f^{j}+f^{j+1}\right) / \tau^{2}\right)+\left(\left(s-t_{j}\right) / \tau\right)\left(\left(f^{j}-2 f^{j+1}+f^{j+2}\right) / \tau^{2}\right)}{\left(t_{n-1+\sigma}-s\right)^{\alpha_{n-1+\sigma}-1}} d s \\
& \left.+\int_{t_{n-1}}^{t_{n-1+\sigma}} \frac{\delta_{t}^{2} f^{n-1}}{\left(t_{n-1+\sigma}-s\right)^{\alpha_{n-1+\sigma}-1}} d s\right]=\frac{1}{\Gamma\left(2-\alpha_{n-1+\sigma}\right)}\left(I_{1}+I_{2}+I_{3}\right) .
\end{aligned}
$$

For the first term in the right hand side of (36), this leads

to

$$
\begin{aligned}
& I_{1}=\int_{t_{0}}^{t_{1}} \frac{\left(\left(s-t_{0}\right) / \tau\right)\left(\left(f^{2}-2 f^{1}+f^{0}\right) / \tau^{2}\right)+\left(\left(t_{1}-s\right) / \tau\right)\left(\left(8 f^{1}-f^{2}-7 f^{0}-6 \tau f_{0}^{\prime}\right) / 2 \tau^{2}\right)}{\left(t_{n-1+\sigma}-s\right)^{\alpha_{n-1+\sigma}-1}} d s \\
& =-\frac{\delta_{t}^{2} f^{1}}{\tau} \int_{t_{0}}^{t_{1}}\left(t_{n-1+\sigma}-s\right)^{2-\alpha_{n-1+\sigma}} d s+(n-1+\sigma) \delta_{t}^{2} f^{1} \int_{t_{0}}^{t_{1}}\left(t_{n-1+\sigma}-s\right)^{1-\alpha_{n-1+\sigma}} d s \\
& +\frac{8 f^{1}-f^{2}-7 f^{0}-6 \tau f_{0}^{\prime}}{2 \tau^{3}} \int_{t_{0}}^{t_{1}}\left(t_{n-1+\sigma}-s\right)^{2-\alpha_{n-1+\sigma}} d s-(n+\sigma-2) \frac{8 f^{1}-f^{2}-7 f^{0}-6 \tau f_{0}^{\prime}}{2 \tau^{2}} \int_{t_{0}}^{t_{1}}\left(t_{n-1+\sigma}-s\right)^{1-\alpha_{n-1+\sigma}} d s \\
& =\frac{\left(3 f^{2}-12 f^{1}+9 f^{0}+6 \tau f_{0}^{\prime}\right) \tau^{-\alpha_{n-1+\sigma}}}{2\left(2-\alpha_{n-1+\sigma}\right)\left(3-\alpha_{n-1+\sigma}\right)}\left[(n+\sigma-1)^{3-\alpha_{n-1+\sigma}}-(n+\sigma-2)^{3-\alpha_{n-1+\sigma}}\right] \\
& +\frac{\left(8 f^{1}-f^{2}-7 f^{0}-6 \tau f_{0}^{\prime}\right) \tau^{-\alpha_{n-1+\sigma}}}{2\left(2-\alpha_{n-1+\sigma}\right)}(n+\sigma-1)^{2-\alpha_{n-1+\sigma}}-\frac{\left(2 f^{2}-4 f^{1}+2 f^{0}\right) \tau^{-\alpha_{n-1+\sigma}}}{2\left(2-\alpha_{n-1+\sigma}\right)}(n+\sigma-2)^{2-\alpha_{n-1+\sigma}} \text {. }
\end{aligned}
$$

For the second term in the right hand side of (36), the following holds:

$$
\begin{aligned}
I_{2} & =\int_{t_{j}}^{t_{j+1}} \frac{\left(\left(t_{j+1}-s\right) / \tau\right)\left(\left(f^{j-1}-2 f^{j}+f^{j+1}\right) / \tau^{2}\right)+\left(\left(s-t_{j}\right) / \tau\right)\left(\left(f^{j}-2 f^{j+1}+f^{j+2}\right) / \tau^{2}\right)}{\left(t_{n-1+\sigma}-s\right)^{\alpha_{n-1+\sigma}-1}} d s \\
& =\frac{\delta_{t}^{2} f^{j}}{\tau} \int_{t_{j}}^{t_{j+1}} \frac{t_{j+1}-s}{\left(t_{n-1+\sigma}-s\right)^{\alpha_{n-1+\sigma}-1}} d s+\frac{\delta_{t}^{2} f^{j+1}}{\tau} \int_{t_{j}}^{t_{j+1}} \frac{s-t_{j}}{\left(t_{n-1+\sigma}-s\right)^{\alpha_{n-1+\sigma}-1}} d s \\
& =\frac{\left(f^{j+2}-3 f^{j+1}+3 f^{j}-f^{j-1}\right) \tau^{-\alpha_{n-1+\sigma}}}{\left(2-\alpha_{n-1+\sigma}\right)\left(3-\alpha_{n-1+\sigma}\right)}\left[(n+\sigma-j-1)^{3-\alpha_{n-1+\sigma}}-(n+\sigma-j-2)^{3-\alpha_{n-1+\sigma}}\right] \\
& +\frac{\left(f^{j+1}-2 f^{j}+f^{j-1}\right) \tau^{-\alpha_{n-1+\sigma}}}{2-\alpha_{n-1+\sigma}}(n+\sigma-j-1)^{2-\alpha_{n-1+\sigma}}-\frac{\left(f^{j+2}-2 f^{j+1}+f^{j}\right) \tau^{-\alpha_{n-1+\sigma}}}{2-\alpha_{n-1+\sigma}}(n+\sigma-j-2)^{2-\alpha_{n-1+\sigma}} .
\end{aligned}
$$

For the third term in the right hand side of (36), this yields

$$
\begin{aligned}
I_{3} & =\int_{t_{n-1}}^{t_{n-1+\sigma}} \frac{\delta_{t}^{2} f^{n-1}}{\left(t_{n-1+\sigma}-s\right)^{\alpha_{n-1+\sigma}-1}} d s \\
& =\frac{\left(f^{n}-2 f^{n-1}+f^{n-2}\right) \sigma^{2-\alpha_{n-1+\sigma}}}{2-\alpha_{n-1+\sigma}} \tau^{-\alpha_{n-1+\sigma}} .
\end{aligned}
$$

Substituting (37)-(39) into (36), we obtain the approximate difference scheme of the ${ }_{0}^{C} D_{t}^{\alpha_{n-1+\sigma}} f\left(t_{n-1+\sigma}\right)$ :

$$
{ }_{0} \Delta_{t}^{\alpha_{n-1+\sigma}} f^{n-1+\sigma}=\frac{\tau^{-\alpha_{n-1+\sigma}}}{\Gamma\left(3-\alpha_{n-1+\sigma}\right)}\left[\sum _ { j = 0 } ^ { n - 2 } a _ { n - j } ^ { ( \alpha _ { n - 1 + \sigma } ) } \left(f^{j+2}\right.\right.
$$




$$
\begin{aligned}
& \left.-2 f^{j+1}+f^{j}\right)+\sum_{j=1}^{n-2} b_{n-j}^{\left(\alpha_{n-1+\sigma}\right)}\left(f^{j+1}-2 f^{j}+f^{j-1}\right) \\
& +\frac{1}{2} b_{n}^{\left(\alpha_{n-1+\sigma}\right)}\left(8 f^{1}-f^{2}-7 f^{0}-6 \tau f_{0}^{\prime}\right) \\
& \left.+\sigma^{2-\alpha_{n-1+\sigma}}\left(f^{n}-2 f^{n-1}+f^{n-2}\right)\right],
\end{aligned}
$$

where

$$
\begin{aligned}
& a_{j}^{\left(\alpha_{n-1+\sigma}\right)}=\frac{1}{3-\alpha_{n-1+\sigma}}\left[(j+\sigma-1)^{3-\alpha_{n-1+\sigma}}\right. \\
& \left.-(j+\sigma-2)^{3-\alpha_{n-1+\sigma}}\right]-(j+\sigma-2)^{2-\alpha_{n-1+\sigma}}, \quad 1 \leq j \leq n, \\
& b_{j}^{\left(\alpha_{n-1+\sigma}\right)}=(j+\sigma-1)^{2-\alpha_{n-1+\sigma}} \\
& -\frac{1}{3-\alpha_{n-1+\sigma}}\left[(j+\sigma-1)^{3-\alpha_{n-1+\sigma}}\right. \\
& \left.\quad-(j+\sigma-2)^{3-\alpha_{n-1+\sigma}}\right], \quad 2 \leq j \leq n-1 .
\end{aligned}
$$

Similar to the proof of the Theorem 2 , the truncation error of formula (40) is given by following theorem.

Theorem 3. For $\alpha_{n-1+\sigma} \in(1,2), f \in C^{4}\left(\left[0, t_{n+1}\right]\right)$, the following holds:

$$
\begin{aligned}
& \left|{ }_{0}^{C} D_{t}^{\alpha_{n-1+\sigma}} f\left(t_{n-1+\sigma}\right)-{ }_{0} \Delta_{t}^{\alpha_{n-1+\sigma}} f^{n-1+\sigma}\right| \\
& \leq \frac{1}{\Gamma\left(3-\alpha_{n-1+\sigma}\right)}\left(t_{n-1+\sigma}^{2-\alpha_{n-1+\sigma}} \max _{t_{0} \leq t \leq t_{n-1}}\left|f^{(4)}(t)\right| \tau^{2}\right. \\
& \left.\quad+c_{2} \sigma^{2-\alpha_{n-1+\sigma}} \max _{t_{n-1} \leq t \leq t_{n}}\left|f^{\prime \prime \prime}(t)\right| \tau^{3-\alpha_{n-1+\sigma}}\right),
\end{aligned}
$$

where $c_{2}$ is a positive constant.

Proof. From (33), we obtain the truncation error

$$
\begin{aligned}
& R^{n-1+\sigma} \\
& \quad=\frac{1}{\Gamma\left(2-\alpha_{n-1+\sigma}\right)}\left[\sum_{j=0}^{n-2} \int_{t_{j}}^{t_{j+1}} \frac{\left(r_{j}^{3}(s)\right)^{\prime \prime}}{\left(t_{n-1+\sigma}-s\right)^{\alpha_{n-1+\sigma}-1}} d s\right. \\
& \left.\quad+\int_{t_{n-1}}^{t_{n-1+\sigma}} \frac{\left(r_{n}^{2}(s)\right)^{\prime \prime}}{\left(t_{n-1+\sigma}-s\right)^{\alpha_{n-1+\sigma}-1}} d s\right] .
\end{aligned}
$$

From Lemma 1, the result below is natural.

$$
\begin{aligned}
{\left[r_{0}^{3}(s)\right]^{\prime \prime}=} & \frac{f^{(4)}\left(\eta_{0}\right)}{2}\left(s-t_{0}^{(0)}\right)\left(s-t_{1}^{(0)}\right), \\
& \eta_{0} \in\left(t_{0}, t_{2}\right), t_{0}^{(0)} \in\left(t_{0}, t_{1}\right), t_{1}^{(0)} \in\left(t_{0}, t_{2}\right), \\
{\left[r_{j}^{3}(s)\right]^{\prime \prime}=} & \frac{f^{(4)}\left(\eta_{j}\right)}{2}\left(s-t_{0}^{(j)}\right)\left(s-t_{1}^{(j)}\right),
\end{aligned}
$$

$$
\begin{array}{r}
\eta_{j} \in\left(t_{j-1}, t_{j+2}\right), t_{0}^{(j)} \in\left(t_{j-1}, t_{j+1}\right), t_{1}^{(j)} \in\left(t_{j}, t_{j+2}\right), \\
{\left[r_{n}^{2}(s)\right]^{\prime \prime}=f^{\prime \prime \prime}\left(\eta_{n}\right)\left(s-t_{0}^{(n)}\right),} \\
\eta_{n} \in\left(t_{n-2}, t_{n}\right), t_{0}^{(n)} \in\left(t_{n-2}, t_{n}\right) .
\end{array}
$$

Substituting (45) into (44), it yields the estimation

$$
\begin{aligned}
& \left|\int_{t_{0}}^{t_{1}} \frac{\left(f^{(4)}\left(\eta_{0}\right) / 2\right)\left(s-t_{0}^{(0)}\right)\left(s-t_{1}^{(0)}\right)}{\left(t_{n-1+\sigma}-s\right)^{\alpha_{n-1+\sigma}-1}} d s\right| \\
& \leq \max _{t_{0} \leq t \leq t_{2}}\left|f^{(4)}(t)\right|\left|\int_{t_{0}}^{t_{1}}\left(t_{n-1+\sigma}-s\right)^{1-\alpha_{n-1+\sigma}} d s\right| \tau^{2} \\
& \leq \frac{t_{n-1+\sigma}^{2-\alpha_{n-1+\sigma}}}{2-\alpha_{n-1+\sigma}} \max _{t_{0} \leq t \leq t_{2}}\left|f^{4}(t)\right| \tau^{2}, \\
& \left|\sum_{j=1}^{n-2} \int_{t_{j}}^{t_{j+1}} \frac{\left(f^{(4)}\left(\eta_{j}\right) / 2\right)\left(s-t_{0}^{(j)}\right)\left(s-t_{1}^{(j)}\right)}{\left(t_{n-1+\sigma}-s\right)^{\alpha_{n-1+\sigma}-1}} d s\right| \\
& \leq 2 \max _{t_{0} \leq t \leq t_{n-1}}\left|f^{(4)}(t)\right|\left|\int_{t_{1}}^{t_{n-1}}\left(t_{n-1+\sigma}-s\right)^{1-\alpha_{n-1+\sigma}} d s\right| \tau^{2} \\
& \leq \frac{t_{n-1+\sigma}^{2-\alpha_{n-1+\sigma}}}{2-\alpha_{n-1+\sigma}} \max _{t_{1} \leq t \leq t_{n-1}}\left|f^{4}(t)\right| \tau^{2} \\
& \left|\int_{t_{n-1}}^{t_{n-1+\sigma}} \frac{f^{\prime \prime \prime}\left(\eta_{n}\right)\left(s-t_{0}^{(n)}\right)}{\left(t_{n-1+\sigma}-s\right)^{\alpha_{n-1+\sigma}-1}} d s\right| \\
& \leq c_{t_{n-1} \leq t \leq t_{n}} \max \left|f^{\prime \prime \prime}(t)\right|\left|\int_{t_{n-1}}^{t_{n-1+\sigma}}\left(t_{n-1+\sigma}-s\right)^{1-\alpha_{n-1+\sigma}} d s\right| \tau
\end{aligned}
$$

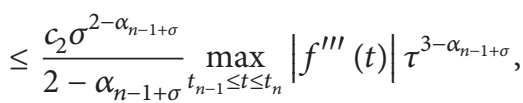

where $c_{2}$ is a positive constant.

Substituting (46) into (44) leads to the theorem.

Remark 4. From the second term of the right hand of the truncation error estimate in Theorem 2, we can easily obtain the fact that formula (14) does not have exact second-order accuracy; it is related to the selection of $\alpha(t)$ and $\sigma$. This can be seen from Table 2 in the numerical example below; its accuracy is much higher than the second order.

\section{Numerical Verification}

In this section, the validity and numerical accuracy of the new presented formula (14) and (40) are demonstrated, respectively. Meanwhile, the corresponding computational results with the formula of [19] are given for contrast. 
TABLE 1: Maximum errors and convergence rates for Example 1 at $T=1$ and $\sigma=1-\alpha_{1} / 2$.

\begin{tabular}{cccccc}
\hline$\alpha(t)$ & $\tau$ & $E^{N}(\tau)(14)$ & Rate $(14)$ & $E^{N}(\tau)[19]$ & $1.726 e-3$ \\
\hline \\
\multirow{2}{*}{$\frac{t^{2}+1}{2}$} & $1 / 40$ & $1.400 e-3$ & 2.0753 & $4.512 e-4$ & 1.9354 \\
& $1 / 80$ & $3.560 e-4$ & 1.9755 & $2.053 e-4$ & 1.9421 \\
& $1 / 120$ & $1.576 e-5$ & 2.0121 & $1.173 e-4$ & 1.9438 \\
& $1 / 160$ & $8.844 e-5$ & 2.0091 & $7.600 e-5$ & 1.9468 \\
\hline
\end{tabular}

TABLE 2: Maximum errors and convergence rates for Example 1 at $T=2$ and $\sigma=1-\alpha_{2} / 2$.

\begin{tabular}{|c|c|c|c|c|c|}
\hline$\alpha(t)$ & $\tau$ & $E^{N}(\tau)(14)$ & Rate (14) & $E^{N}(\tau)$ & Rate [19] \\
\hline \multirow{5}{*}{$e^{-t}$} & $1 / 40$ & $5.830 e-4$ & 2.5859 & $4.834 e-2$ & 1.9724 \\
\hline & $1 / 80$ & $9.138 e-5$ & 2.6734 & $1.232 e-2$ & 1.9843 \\
\hline & $1 / 120$ & $3.021 e-5$ & 2.7145 & $5.509 e-3$ & 1.9889 \\
\hline & $1 / 160$ & $1.362 e-5$ & 2.7462 & $3.109 e-3$ & 1.9914 \\
\hline & $1 / 200$ & $7.285 e-6$ & - & $1.993 e-3$ & - \\
\hline
\end{tabular}
denote

Take a positive integer $N$, let $T=t_{n-1+\sigma}, \tau=T / N$, and

$$
\begin{aligned}
F^{n-1+\sigma} & ={ }_{0}^{C} D_{t}^{\alpha_{n-1+\sigma}} f\left(t_{n-1+\sigma}\right), \\
f^{n-1+\sigma} & ={ }_{0} \Delta_{t}^{\alpha_{n-1+\sigma}} f^{n-1+\sigma}, \\
E^{N}(\tau) & =\left|F^{n-1+\sigma}-f^{n-1+\sigma}\right|, \\
\text { Rate } & =\log _{2} \frac{E^{N}(\tau)}{E^{N}(\tau / 2)} .
\end{aligned}
$$

Example 1 (accuracy of formula (14) and [19]). Take $f(t)=$ $t^{5}-t^{3}, 0<t \leq T$. Compute the Caputo fractional derivative of $f(t)$ at $T=1,2$ numerically.

The exact solution is given by

$$
\begin{aligned}
{ }_{0}^{C} D_{t}^{\alpha_{n-1+\sigma}} f\left(t_{n-1+\sigma}\right)= & \frac{\Gamma(6)}{\Gamma\left(6-\alpha_{n-1+\sigma}\right)} t^{5-\alpha_{n-1+\sigma}} \\
& -\frac{\Gamma(4)}{\Gamma\left(4-\alpha_{n-1+\sigma}\right)} t^{3-\alpha_{n-1+\sigma}} .
\end{aligned}
$$

From the results presented in Table 1, taking $\sigma=$ $1-\alpha_{1} / 2=1 / 2$, we found that although both formula (14) and [19] have second-order approximation accuracy, the numerical accuracy by the formula (14) is significantly higher than that by the formula of [19]; furthermore, the computational errors are also obviously smaller than the formula of [19]. Thus, the new formula (14) is valid for solving complex problems that require high accuracy, and better computational results can be obtained from the formula.

In Table 2, taking $\sigma=1-\alpha_{2} / 2=0.9323$, it shows that the convergence order is higher as compared with the theoretical result, and the error is smaller than the theoretical result, but why this happens is not clear yet; it requires further investigation.

Moreover, on the basis of Table 1, if a slight disturbance to $\sigma$ is added to Tables 3 and 4 , the experimental results will be greatly different. It can be easily found that there is a very close relationship between the convergence order and the selection of $\sigma$. The experimental results show that the convergence order is the ideal result only when $\sigma=1-\alpha_{1} / 2$. Tables 2 , 5 , and 6 also illustrate this fact.

Example 2 (accuracy of formula (40)). Take $f(t)=t^{5}$, $f^{\prime}(0)=0,0<t \leq T$. Compute the Caputo fractional derivative of $f(t)$ at $T=1$ numerically.

The exact solution is given by

$$
{ }_{0}^{C} D_{t}^{\alpha_{n-1+\sigma}} f\left(t_{n-1+\sigma}\right)=\frac{\Gamma(6)}{\Gamma\left(6-\alpha_{n-1+\sigma}\right)} t^{5-\alpha_{n-1+\sigma}} .
$$

Table 7 lists the maximum errors and convergence rates for the variable-order $\sin (t)+1$. In particular, when $T=1$, $\alpha\left(t_{n-1+\sigma}\right)=\sin (1)+1=1.8415$, and $\sigma=0.5793$, the numerical results demonstrate that formula (40) leads to 3 $\alpha(t)$ accuracy.

Similar to Example 1, we also give Tables 8 and 9 as a comparison; the results show that $\sigma=3 / 2-\alpha_{1} / 2$ is the best choice.

Remark 3. Due to the fact that the selection of $\sigma$ is very important, it is worth noting that different selected $\sigma$ leads to the different numerical accuracy. From considerable trials, we observed that only if we take $\sigma=1-\alpha(t) / 2$ for $\alpha(t) \in(0,1)$ and $\sigma=3 / 2-\alpha(t) / 2$ for $\alpha(t) \in(1,2)$, will numerical results be closer to theoretical analysis. In addition, carefully observing Table 9, although the convergence order is not higher than in Table 7, the error is lower than in Table 7; further investigation is needed on this phenomenon.

\section{Conclusion}

In this paper, two approximations to variable-order Caputo fractional derivatives were developed, and the analysis for the truncation errors of new formulas was made. In addition, numerical examples support theoretical results. In further work, we also want to give a strict proof of the selection of 
TABLE 3: Maximum errors and convergence rates for Example 1 at $T=1$ and $\sigma_{1}=\sigma-0.1$.

\begin{tabular}{cccc}
\hline$\alpha(t)$ & $\tau$ & $E^{N}(\tau)$ & Rate \\
\hline & $1 / 40$ & $3.720 e-2$ & 1.0847 \\
$\frac{t^{2}+1}{2}$ & $1 / 80$ & $1.800 e-2$ & 1.0473 \\
& $1 / 120$ & $1.190 e-2$ & 1.0300 \\
& $1 / 160$ & $8.900 e-3$ & 1.0161 \\
$1 / 200$ & $7.100 e-3$ & 1.0101 \\
\hline
\end{tabular}

TABLE 4: Maximum errors and convergence rates for Example 1 at $T=1$ and $\sigma_{2}=\sigma+0.1$.

\begin{tabular}{cccr}
\hline$\alpha(t)$ & $\tau$ & $E^{N}(\tau)$ & Rate \\
\hline & $1 / 40$ & $3.380 e-2$ & 0.9412 \\
$\frac{t^{2}+1}{2}$ & $1 / 80$ & $1.720 e-2$ & 0.9746 \\
& $1 / 120$ & $1.150 e-2$ & 0.9874 \\
& $1 / 160$ & $8.700 e-3$ & 0.9833 \\
& $1 / 200$ & $7.000 e-3$ & 0.9792 \\
\hline
\end{tabular}

TABle 5: Maximum errors and convergence rates for Example 1 at $T=2$ and $\sigma_{3}=\sigma+0.1$.

\begin{tabular}{cccc}
\hline$\alpha(t)$ & $\tau$ & $E^{N}(\tau)$ & Rate \\
\hline & $1 / 40$ & $3.070 e-2$ & 1.8040 \\
& $1 / 80$ & $8.600 e-3$ & 1.8358 \\
$e^{-t}$ & $1 / 120$ & $4.100 e-3$ & 1.8323 \\
& $1 / 160$ & $2.400 e-3$ & 1.8413 \\
& $1 / 200$ & $1.600 e-3$ & 1.8329 \\
\hline
\end{tabular}

TABLE 6: Maximum errors and convergence rates for Example 1 at $T=2$ and $\sigma_{4}=\sigma-0.23$.

\begin{tabular}{cccc}
\hline$\alpha(t)$ & $\tau$ & $E^{N}(\tau)$ & Rate \\
\hline & $1 / 40$ & $5.390 e-2$ & 1.8808 \\
& $1 / 80$ & $1.470 e-2$ & 1.8745 \\
$e^{-t}$ & $1 / 120$ & $6.900 e-3$ & 1.8688 \\
& $1 / 160$ & $4.000 e-3$ & 1.8777 \\
& $1 / 200$ & $2.600 e-3$ & 1.8995 \\
\hline
\end{tabular}

TABle 7: Maximum errors and convergence rates for Example 2 at $T=1$ and $\sigma=3 / 2-\alpha_{1} / 2$.

\begin{tabular}{lccr}
\hline$\alpha(t)$ & $\tau$ & $E^{N}(\tau)$ & Rate \\
\hline & $1 / 40$ & $4.023 e-1$ & 1.1310 \\
& $1 / 80$ & $1.821 e-1$ & 1.1435 \\
$\sin (t)+1$ & $1 / 160$ & $8.210 e-2$ & 1.1493 \\
& $1 / 320$ & $3.690 e-2$ & 1.1538 \\
& $1 / 640$ & $1.660 e-2$ & 1.1524 \\
& $1 / 1280$ & $7.400 e-3$ & 1.1656 \\
\hline
\end{tabular}

TABle 8: Maximum errors and convergence rates for Example 2 at $T=1$ and $\sigma_{5}=\sigma+0.2$.

\begin{tabular}{lccc}
\hline$\alpha(t)$ & $\tau$ & $E^{N}(\tau)$ & Rate \\
\hline & $1 / 40$ & $5.644 e-1$ & 1.1219 \\
& $1 / 80$ & $2.562 e-1$ & 1.1394 \\
$\sin (t)+1$ & $1 / 160$ & $1.156 e-1$ & 1.1481 \\
& $1 / 320$ & $5.200 e-2$ & 1.1526 \\
& $1 / 640$ & $2.33 e-2$ & 1.1582 \\
& $1 / 1280$ & $1.050 e-2$ & 1.1499 \\
\hline
\end{tabular}


TABle 9: Maximum errors and convergence rates for Example 2 at $T=1$ and $\sigma_{6}=\sigma-0.3$.

\begin{tabular}{lccr}
\hline$\alpha(t)$ & $\tau$ & $E^{N}(\tau)$ & Rate \\
\hline & $1 / 40$ & $1.705 e-1$ & 1.1216 \\
& $1 / 80$ & $7.760 e-2$ & 1.1356 \\
$\sin (t)+1$ & $1 / 160$ & $3.510 e-2$ & 1.1446 \\
& $1 / 320$ & $1.580 e-2$ & 1.1515 \\
& $1 / 640$ & $7.100 e-3$ & 1.1540 \\
& $1 / 1280$ & $3.200 e-3$ & 1.1497 \\
\hline
\end{tabular}

$\sigma$ and apply these new methods to solve more complex cases within a reasonable accuracy.

\section{Conflicts of Interest}

The authors declare that there are no conflicts of interest regarding the publication of this paper.

\section{Acknowledgments}

This work is supported by Science and Technology Department Key Projects of Fujian Province, China (Grant 2014H0034), and the Natural Science Foundation of Fujian Province, China (Grants 2017J01402, 2017J01557, and 2016J01310).

\section{References}

[1] S. G. Samko and B. Ross, "Integration and differentiation to a variable fractional order," Integral Transforms and Special Functions, vol. 1, no. 4, pp. 277-300, 1993.

[2] S. G. Samko, "Fractional integration and differentiation of variable order," Analysis Mathematica, vol. 21, no. 3, pp. 213-236, 1995.

[3] C. F. Lorenzo and T. T. Hartley, "Variable order and distributed order fractional operators," Nonlinear Dynamics, vol. 29, no. 1-4, pp. 57-98, 2002.

[4] S. Samko, "Fractional integration and differentiation of variable order: an overview," Nonlinear Dynamics, vol. 71, no. 4, pp. 653662, 2013.

[5] C. F. Coimbra, "Mechanics with variable-order differential operators," Annalen der Physik, vol. 12, no. 11-12, pp. 692-703, 2003.

[6] C. M. Soon, C. F. M. Coimbra, and M. H. Kobayashi, "The variable viscoelasticity oscillator," Annalen der Physik, vol. 14, no. 6, pp. 378-389, 2005.

[7] X. Li and B. Wu, "A numerical technique for variable fractional functional boundary value problems," Applied Mathematics Letters, vol. 43, pp. 108-113, 2015.

[8] H. Sheng, H. G. Sun, C. Coopmans, Y. Q. Chen, and G. W. Bohannan, "A Physical experimental study of variable-order fractional integrator and differentiator," The European Physical Journal: Special Topics, vol. 193, no. 1, pp. 93-104, 2011.

[9] H. Sun, W. Chen, and Y. Chen, "Variable-order fractional differential operators in anomalous diffusion modeling," Physica A: Statistical Mechanics and Its Applications, vol. 388, no. 21, pp. 4586-4592, 2009.

[10] H. G. Sun, W. Chen, H. Wei, and Y. Q. Chen, "A comparative study of constant-order and variable-order fractional models in characterizing memory property of systems," The European Physical Journal: Special Topics, vol. 193, no. 1, pp. 185-192, 2011.

[11] J. Cao and Y. Qiu, "A high order numerical scheme for variable order fractional ordinary differential equation," Applied Mathematics Letters, vol. 61, pp. 88-94, 2016.

[12] Z.-J. Fu, W. Chen, and L. Ling, "Method of approximate particular solutions for constant- and variable-order fractional diffusion models," Engineering Analysis with Boundary Elements, vol. 57, pp. 37-46, 2015.

[13] R. Lin, F. Liu, V. Anh, and I. Turner, "Stability and convergence of a new explicit finite-difference approximation for the variable-order nonlinear fractional diffusion equation," Applied Mathematics and Computation, vol. 212, no. 2, pp. 435-445, 2009.

[14] C.-M. Chen, F. Liu, V. Anh, and I. Turner, "Numerical schemes with high spatial accuracy for a variable-order anomalous subdiffusion equation," SIAM Journal on Scientific Computing, vol. 32, no. 4, pp. 1740-1760, 2010.

[15] S. Shen, F. Liu, J. Chen, I. Turner, and V. Anh, "Numerical techniques for the variable order time fractional diffusion equation," Applied Mathematics and Computation, vol. 218, no. 22, pp. 10861-10870, 2012.

[16] C.-M. Chen, F. Liu, V. Anh, and I. Turner, "Numerical methods for solving a two-dimensional variable-order anomalous subdiffusion equation," Mathematics of Computation, vol. 81, no. 277, pp. 345-366, 2012.

[17] H. Zhang, F. Liu, M. S. Phanikumar, and M. M. Meerschaert, "A novel numerical method for the time variable fractional order mobile-immobile advection-dispersion model," Computers \& Mathematics with Applications, vol. 66, no. 5, pp. 693-701, 2013.

[18] C.-M. Chen, F. Liu, I. Turner, V. Anh, and Y. Chen, "Numerical approximation for a variable-order nonlinear reactionsubdiffusion equation," Numerical Algorithms, vol. 63, no. 2, pp. 265-290, 2013.

[19] X. Zhao, Z.-z. Sun, and G. E. Karniadakis, "Second-order approximations for variable order fractional derivatives: algorithms and applications," Journal of Computational Physics, vol. 293, pp. 184-200, 2015.

[20] A. A. Alikhanov, "A new difference scheme for the time fractional diffusion equation," Journal of Computational Physics, vol. 280, pp. 424-438, 2015. 


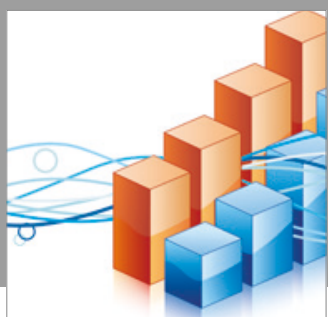

Advances in

Operations Research

vatersals

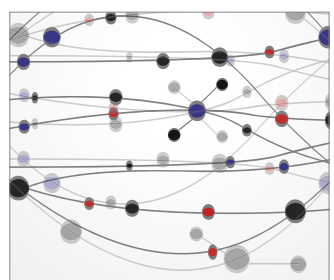

\section{The Scientific} World Journal
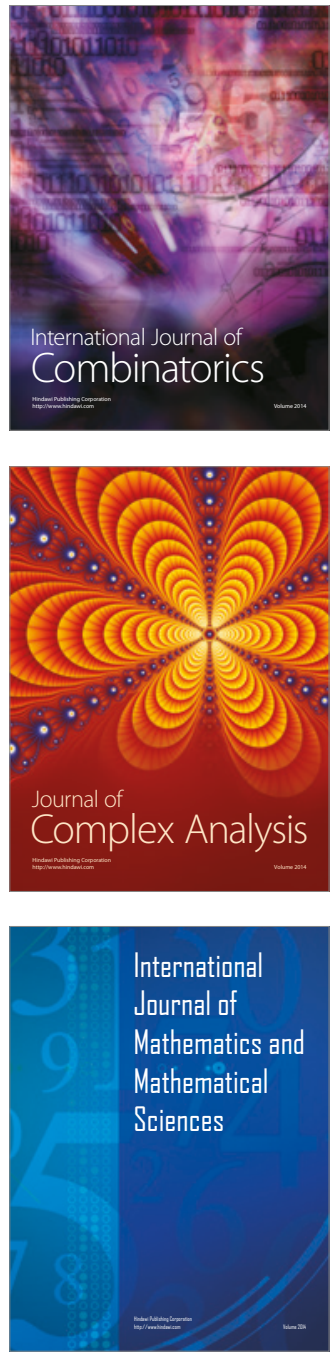
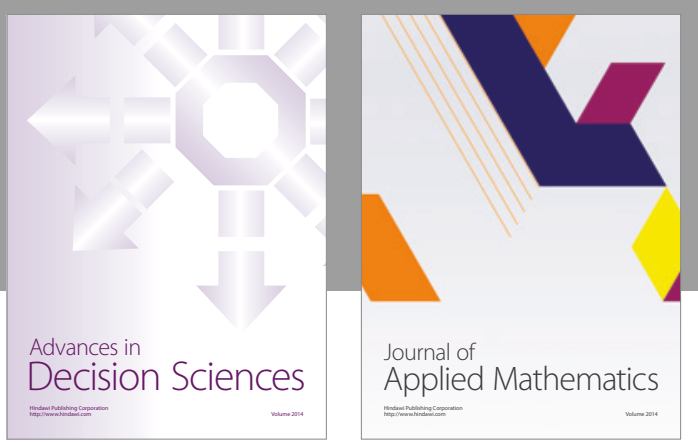

Algebra

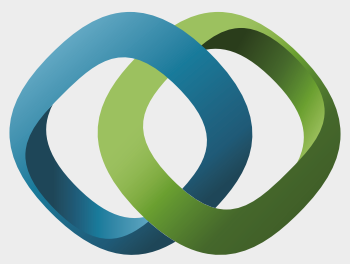

\section{Hindawi}

Submit your manuscripts at

https://www.hindawi.com
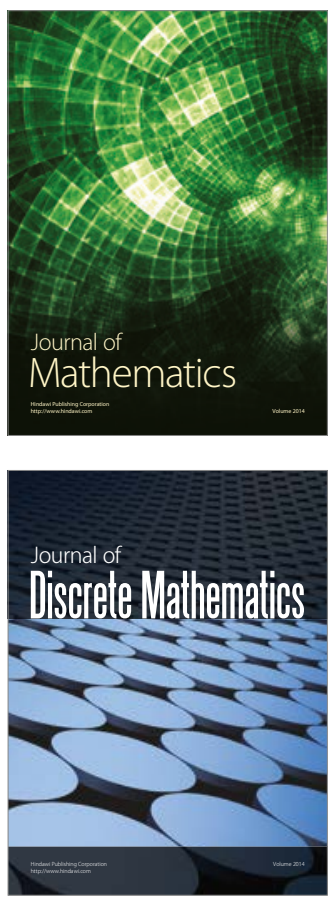

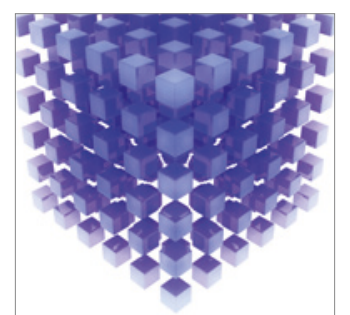

Mathematical Problems in Engineering
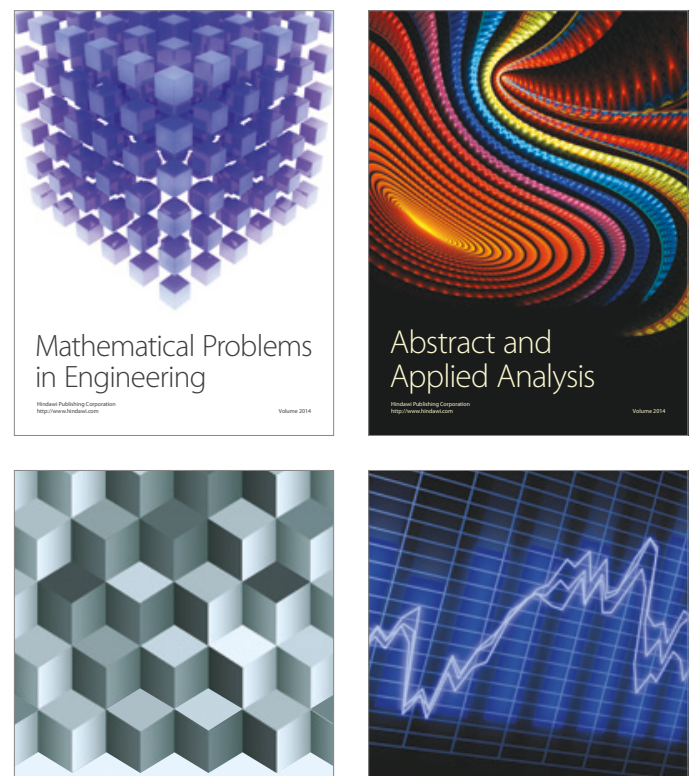

Journal of

Function Spaces

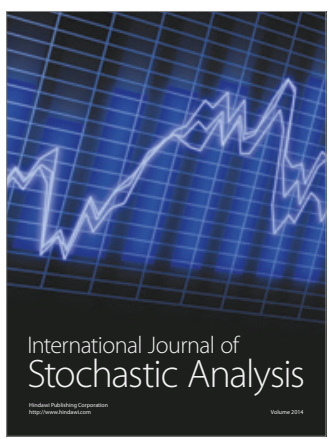

Probability and Statistics
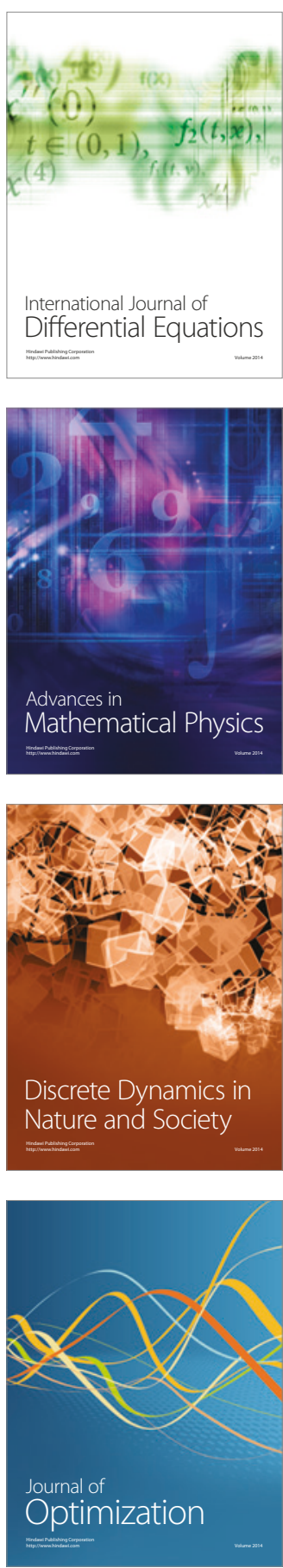